\title{
Whither the acute medical patient?
}

\author{
T D Wardle
}

\begin{abstract}
The continual change in NHS structure demands reappraisal of both the services provided and the allocation of resources to ensure appropriate standards of care. Unfortunately this equation never seems to balance. Although the overall goal is to have an excellent standard of care, the necessary resources nearly always are lacking. As a consequence "we" often have to critically analyse and change clinical practice to achieve this goal. Nowhere in medicine is this point more pertinent than in the management of acute medical emergencies. This article examines not only how the changing face of medicine influences this situation but also possible solutions to the question who will manage these patients in the future.

( $P$ Accid Emerg Med 1997;14:167-169)
\end{abstract}

Keywords: NHS structure; future policy; management of acute medical emergencies

It is with great regret that we announce the passing of the general physician. No flowers please, donations to medical charities. ${ }^{1}$

A surprise? Not really, like many fine thoroughbred species in demand, their members become culled by the savage beast-the changing health service. Evolution is usually a beneficial process which strengthens positive characteristics and reduces weakness. In doing so it enables the species to survive and facilitates development-but is this always the case?

The physician is often regarded as the father of medicine, originating in Sumeria in approximately $3000 \mathrm{BC}^{2}$ Physicians were deemed of priestly class with primitive magical and religious ideas dominating their medical concepts. Many still acknowledge that while Aesculapius was regarded as the God of Medicine, Hippocrates was the Father. ${ }^{3}$ Various cultures contributed widely differing concepts to the development of medicine but English physicians had little influence until the 13th century. Subsequently, in 1518, Thomas Linacre gained royal consent to form a regular body of physicians that became the Royal College of Physicians in 1551.

Although different subspecialties were arising in surgery and pathology, the process was gradual and less obvious with general medicine. In the middle of the 19 th century definitive evidence of future trends was manifest as neurology became a distinct branch of medicine. Subsequently, cardiology arose out of physiology and coincided with the emergence of dermatology and venereology as specialities in their own right.

When did the pages of the medical journals last contain adverts for a general physician? Current jobs are often advertised as "General Physician with a specialist interest in ..." and most colleagues will call themselves, for example, a Consultant Gastroenterologist; is this denial of their role or a reflection of the future? If either or both of these statements are true could medicine cope without the general physician? Who, if anybody, could replace this doyen of diagnosis? Overriding these, however, is the all important question-is the general physician a threatened species? Sadly the answer is yes!

Why? Superspecialisation is the problem. With rapid advances in all disciplines of internal medicine many current, and embryonic, consultants are becoming blinkered. As a result they are dedicating most of their time to their super specialist interest at the expense of general medicine. Some specialist groups, in particular the neurologists and cardiologists, have led the defection either partially or totally. What is even more worrying is that recent surveys suggest more general physicians, especially in gastroenterology and cardiology, would like to follow suit. ${ }^{4}$ What is, therefore, to become of the emergency general medical patient? Who will be responsible for acute medical admissions, especially when faced with an increase in the number of acute patients combined with the ever present reduction in the number of beds?

Four potential systems of organising care offer potential solutions to these questions. Each system merits consideration as it is unlikely that one will suit the needs of all acute medical units. The final choice will be influenced by, and have to be tailored to, the type and size of the hospital along with the resources, including staff, that are available.

The four systems are:

(1) Ensure that consultant physicians oversee the management of acute medical patients on either daily or weekly rotation. 
(2) Create a new discipline within internal medicine-a consultant physician with an interest in acute medicine.

(3) Dovetail the management of acute medical patients into other specialties to produce hybrid specialists, for example, with emergency medicine and/or intensive care.

(4) Dispense with the emergency take and triage appropriate patients to the specialty ward.

Whatever the chosen model, consultants must lead the service and they should review patients within a maximum of 24 hours after admission. Moreover this must be achieved despite the recently imposed constraints affecting junior doctors' hours and consultants' workload. Although the changes in junior doctors' hours have ensured that their number is increasing, they spend less time "on the shop floor" because of the combination of a reduction in hours and more structured training. In contrast, consultants are under increasing demands from not only the government but also the purchasers and the patients. The government expect greater efficiency. The purchasers want consultants to provide more "hands on care" especially for acute medical emergencies. The patients have increasing expectations of service provision. Furthermore, consultant physicians are also required to reduce out patient waiting times, audit their activities, increase contribution to managerial activities, ensure adequate $\mathrm{CME}$, teaching and possibly research, as well as come to terms with the increasing complexity and frequency of innovations, not only in their specialist field but also in medicine as a whole. The net effect is an increase in consultants' work load. Thus we have to be proactive in organising the delivery of care and ensure protected time for these activities including the management of acute medical emergencies, especially as they occupy approximately $90 \%$ of medical beds. ${ }^{5}$

With these constraints in mind the salient points of each care system will be outlined.

System (1)-Ensure that consultant physicians control the acute medical take by one of the following:

(a) Individual consultants providing daily cover with at least two ward rounds per day

(b) Two consultants sharing the daily intake, either in 12 hour shifts or accepting for alternate groups of, for example, four patients. These patients would be admitted to either the dedicated consultant's ward or an admission ward.

(c) Individual consultants, rostered, to oversee admissions for five days from 0800 to 1800 with colleagues doing a ward round each evening (2100) and twice at weekends (0800, 2000). Specialist colleagues would be requested to consult over difficult diagnostic or management problems. This system requires a central "admission ward" from where patients will either be discharged or triaged to the appropriate specialist ward.

Thus, regular ward rounds and planned discharges would facilitate the smooth running of the ward as well as ensure optimum bed use and prevent colleagues from being swamped by non-specialist patients. ${ }^{6}$ The consultant would also be available to liaise with colleagues in primary care and discuss management problems and potential admissions. If, however, admission was not warranted then advice would be given regarding the best environment for patient care, that is, rapid access clinics, outpatients, home, nursing home, or hospice.

System (2)-A physician with a specialist interest in acute medicine, or one who is solely dedicated to the care of emergency patients. The physician would look after patients with all acute medical problems, including myocardial infarction, gastrointestinal haemorrhage, and those requiring nasal CPAP. The proximity, or integration, of wards allocated for medical admissions, high dependency care, and coronary care would facilitate the development of this position.

The acute medical physician would use other specialist consultant colleagues as a resource, requesting a consultation-in the true meaning of the word-if the need was dictated by the clinical situation. This is particularly prudent as specialist care is better than that provided by a generalist. ${ }^{7}$ If admission is deemed necessary by the consultant, then the patient would receive acute medical care, after which they would either be discharged or triaged to the relevant specialist ward as described earlier.

The logistics of a rota would have to be negotiated with colleagues in either internal medicine, intensive care, or the emergency department, or a combination of these. This interdepartmental cooperation would be mandatory as it is unlikely that sufficient funding would be available to enable a group of specialists within the new specialty to operate a " 38 hours on, five days off" rota.

General medical training to MRCP level would still be necessary but with further experience in intensive care, anaesthetics, and emergency medicine.

More specialists are needed, but with current finances this would be deemed wasteful. An alternative would be to concentrate more specialists in fewer hospitals, but this may induce problems with patient transport and with contracts between health authorities. Job satisfaction could be excellent, with a rota similar to some emergency medicine departments. Unfortunately, this is unlikely to occur. Consequently, the next option, "a hybrid specialist", should be considered.

System (3)-Dovetail the management of acute medical patients into other specialties. The development and recognition of both accident and emergency medicine and intensive care has enhanced the management of acute medical emergencies. The benefits of general medical training are exemplified, in part, by the fact that candidates with MRCP are recruited into these specialties. Furthermore the educational needs of future intensivists have been recently recognised, and hence the development of the intercollegiate committee on training in intensive care medicine. Thus it would seem logical to devolve the management of acute medical patients to these specialties. This would have a 
major impact on training as more experience in general medicine would be required.

Consultants within these disciplines are unlikely to accept further responsibility as they are already scarce and overworked. Moreover, many acute medical patients would not require specialist resuscitation/intensivist skills. Thus the integration of acute medicine with emergency medicine and intensive care may have a negative effect on recruitment for all disciplines involved.

System (4)-Dispense with the emergency intake and triage patients to the appropriate specialty ward. Triage would be done by the general practitioner on referral. While this system would be ideal during normal working hours, it would create havoc with the on call admitting staff and would mean that either one consultant or a representative from a team of consultants from each specialty would have a daily or twice daily ward round. Who would care for patients following overdose or with a pyrexia of unknown origin? Although the numbers of patients admitted per ward will be small, with seasonal variation, this duty would be onerous and have a major impact on other aspects of the consultant's workload. Furthermore, some specialists would receive a disproportionate number of patients, as the most frequent reasons for admission are myocardial infarction, cardiac failure, acute exacerbations of asthma and chronic airflow limitation, stroke, and drug overdose. ${ }^{89}$ With current fluctuations in budgets and staff numbers this option would not be feasible without considerable changes in the delivery of care.

Irrespective of the system used to manage acute medical patients, the common denominator is that "more specialist consultants are required". However, consultants' posts are difficult enough to fill, so how will recruitment for either new consultants in acute medicine or those with an interest in this discipline be viewed? Furthermore, will the more senior physicians delight in managing acute medical patients? The later is potentially a major disincentive, but not for the recently appointed consultant. Therefore, to facilitate consultants' development it is conceivable that after a number of years they could opt out of managing acute medical patients and focus, for example, on management issues.

Whatever the definitive model of acute medical care the answer is to protect or reincarnate the general physician. This has even been acknowledged in the home of superspecialisation, the USA. The exodus from gen- eral to pure specialist physicians must be stopped or at least reduced, but how? The reputation of general internal medicine needs to be enhanced by both the Royal College of Physicians and the NHS executive. Initial general "MRCP" training should continue, followed by a specific training structured to ensure a balance between acute/emergency medicine and the chosen specialty. Furthermore, to facilitate development of this career pathway recognition by medical colleagues and the NHS executive is mandatory. Resources are also required, without conflict with other specialities, to ensure that well motivated enthusiastic consultants find the challenge of general medicine attractive. This, in turn, will enable advances in both disciplines, culminating in innovative methods of managing the acute medical patient.

Thus the face of acute medicine is changing. Furthermore, the number of acute medical admissions is increasing. We have to be proactive and decide how we can deliver care within current resource constraints rather than respond reactively, too late, to a situation that will certainly deteriorate. This is a universal problem that will continue to have an impact upon staff in both general and emergency medicine. Therefore, within each hospital, specialists from both of these disciplines need to liaise and critically examine their current admission strategies to provide optimum management of acute medical emergencies. Moreover, we should look to our respective Royal College and Faculty to develop a committee that will ensure appropriate training in this important medical discipline. Despite the potential demise of the general physician and the changing health service there is one constant, ever present, factor-the general medical patient!

1 Anon. The passing of the general physician. BMJ 1999;1:1 2 Smith S. Early history of Assyria to 1000 BC. London: Chatto and Windus, 1928.

3 Guthrie G. The history of medicine. London: Thomas Nelson and Sons, 1958.

4 Farthing $M$, Walt R, Allan RN, Swan CHJ, Gilmore IT, Mallinson $\mathrm{CN}$, et al. A national training programme for gastroenterology and hepatology. Gut 1996;38:459-70.

5 Audit Commission. Lying in wait. London: HMSO, 1991.

6 Department of Health. Discharge of patients from hospital. Department of Health. Disc

7 Bucknall C. Differences in hospital asthma management. Lancet 1988;i:748-50.

8 Bakhai A, Goodman F, Juchniewichz H, et al. How easy is it to contact the duty medical doctor responsible for acute admissions? BMJ 1990;301:529-3

9 Lemrow N, Adams D, Coffey R, Farley D. The 50 most frequent diagnosis-related groups (DRGs), diagnoses and procedures: statistics by hospital size and location. DHSS Publication No (PHS) 90-3465. Hospital Studies Program Research 'Note 13, Agency for Health Care Policy and Research, Rockville, MD: Public Health Service. 